\title{
A 350-MS/s Continuous-Time Delta-Sigma Modulator with a Digitally-Assisted Binary-DAC and a 5-Bits Two-Step-ADC Quantizer in 130-nm CMOS
}

\author{
Mohammad Taherzadeh-Sani and Frederic Nabki
}

\begin{abstract}
Two techniques to improve the performance of continuous-time delta-sigma modulators are presented. A digital calibration technique is introduced to enable the use of binary current digital-to-analog converters (DAC) without dynamic element matching (DEM). Furthermore, a high-speed two-stepADC quantizer is introduced to efficiently increase the resolution of the quantizer in continuous-time delta-sigma modulators with high-sampling rates. A proof-of-concept prototype implemented in 130-nm CMOS shows that the proposed calibration technique can compensate for up to $5 \%$ of mismatch in the DAC elements. The modulator has a measured SNDR / SFDR of $60.3 \mathrm{~dB} / 74 \mathrm{~dB}$ for a sampling rate of $350 \mathrm{MS} / \mathrm{s}$ and oversampling ratio (OSR) of 20, translating to an $8.75 \mathrm{MHz}$ bandwidth. The total power consumption is $5.5 \mathrm{~mW}$ from a $1.6 \mathrm{~V}$ supply.
\end{abstract}

Index Terms - Delta-sigma modulator, continuous-time, selfcalibration, two-step ADC

\section{INTRODUCTION}

$\mathrm{C}$ ontinuous-time delta-sigma (CTDS) modulators are an

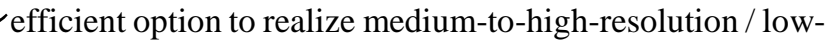
to-medium-bandwidth analog-to-digital data converters (ADCs). Current-mode digital-to-analog converters (DACs) are widely used as one of the building blocks in CTDS modulators. These DACs must be extremely linear to attain sufficient linearity for the modulator. Conventionally, single-bit DACs that are inherently linear are utilized in delta-sigma modulators. However, they are sensitive to clock jitter [1]. Furthermore, in single-loop delta-sigma modulators with high integrator gain to maximize the signal to noise ratio (SNR), they can result in an unstable output [2]-[4]. Accordingly, multi-bit DACs are required to attain less sensitivity to clock jitter [1] and stable operation with a good SNR in high-order modulators.

The inherent current-cell mismatches in the current-mode DACs of a multi-bit structure limit the linearity performance of the CTDS modulator. To mitigate this problem, dynamic element matching (DEM) techniques are usually utilized to shape the DAC element mismatch errors to high frequencies. A drawback of DEM is that the required shuffling of all DAC elements dramatically increases the circuit complexity as a function of the number of involved current cells [5]. This results in an excess loop delay (ELD) in the modulator's feedback path which can cause the DAC pulse to be shifted into the next clock cycle. This effectively increases the order of the loop filter, potentially de-stabilizing the modulator and degrading its noiseshaping performance, particularly in high-speed CTDS

M. Taherzadeh-Sani is with the Electrical Engineering Department, Ferdowsi University of Mashhad, Mashhad, Iran (email: Taherzadeh@um.ac.ir). F. Nabki is with CoFaMic Research Centre, Université du Québec à Montréal, Montréal, Québec, Canada. modulators [6, 7].

A promising technique to substitute the DEM block is DAC calibration. There are limited reported works on DAC calibration in CTDS modulators [2], [8]-[11]. These techniques are reported for unary DAC structures. Thus, the complexity of these techniques (e.g., the number of switches and their control signals, the number of unit element in the layout, etc.) can significantly increase when applying the technique in a higher resolution DAC.

This paper proposes a digital DAC self-calibration technique that can be used in binary current-mode DACs. The proposed calibration technique is a foreground method and requires no significant additional analog circuitry. The digital correction block used to implement the calibration is also very simple due to the limited number of correction coefficients necessary for the binary output of the modulator.

Traditionally, delta-sigma modulators utilize a unary DAC architecture, where the complexity is dramatically increased by the DAC resolution. However, the proposed DAC calibration allows the proposed CTDS modulator to employ a binary DAC architecture without DEM. Thus, due to the simplicity of the binary DAC cell, increasing DAC resolution becomes practical, and the resolution of the modulator's quantizer is also increased. Although flash ADCs are the common architecture to realize the quantizer, they require many comparators and a relatively large encoder, complicating the design of the quantizer. Alternatively, a two-step ADC quantizer is a good candidate to increase the quantizer's resolution without requiring many comparators and a large digital encoder (e.g., $[12,13]$ in discrete-time modulators). Thus, in this work, a multi-bit two-step-ADC quantizer, along with a foreground digital binary-DAC calibration technique, is introduced within the continuous-time delta-sigma modulator.

As a proof-of-concept, a $2^{\text {nd }}$-order CTDS modulator that utilizes the proposed techniques was implemented in $130 \mathrm{~nm}$ CMOS technology. Section 2 presents the delta-sigma modulator architecture, while Section 3 details the two-stepADC quantizer. In section 4, the binary-DAC calibration technique is proposed and detailed. Finally, Section 5 presents the measurement results of the implemented CTDS modulator.

\section{Delta-Sigma Modulator ARChitecture}

The key contributions of this work are $i$ ) the DAC calibration technique that mitigates the need for DEM, and ii) the two-stepADC that allows for increased quantizer resolution. As such, a conventional $2^{\text {nd }}$-order feedforward continuous-time loop filter 
is employed in the CTDS modulator, as shown in Fig. 1.

In the loop-filter of the modulator, the integrators are realized using opamp-based RC integrators. The adder in front of the quantizer is an opamp-based resistive adder. All opamps have a telescopic structure to minimize their power and enhance their speed. The input is also directly added to the quantizer input in order to minimize the swing requirement of the adder's opamp. Note that this addition is performed directly at the quantizer after the adder, as seen in Fig. 1. Figure 2 shows the circuit details of the loop filter. The two-step ADC quantizer has a 5bit resolution with one redundant bit, as described in Section 3. The conventional feedback path is closed through DAC1, which is a binary current non-return-to-zero (NRZ) DAC that is calibrated as detailed in Section 4.

The excess loop delay which is a timing delay in the feedback DAC pulse results from the finite time required for the twostep-ADC quantizer to resolve its input and for the DAC1 to respond to the ADC output. This timing delay can cause the DAC pulse to be shifted into the next clock cycle which can potentially de-stabilize the modulator and degrade its noiseshaping performance [6, 7]. Here, the excess loop delay can be compensated for by modifying the feedforward coefficients and introducing an additional feedback path through DAC2 from the modulator output to the input of the quantizer, as shown in Fig. 1 [7]. DAC2 is also a binary current NRZ DAC, but it requires no calibration, as its errors are shaped by the loop filter in a similar fashion to the well-known shaping of quantization noise in the modulator.

In the primary design of the modulator, the equivalent discrete-time noise transfer function (NTF) of this $2^{\text {nd }}$-order CTDS modulator is considered to be:

$$
\operatorname{NTF}(z)=\left(1-z^{-1}\right)^{2}
$$

The impulse-invariant transform is used to synthesize the equivalent continuous-time transfer function of this NTF [6]. A methodology for determining the feedforward coefficients and the additional feedback coefficient through DAC2 is presented in [6].

\section{TWO-STEP ADC QUANTIZER}

Flash ADCs are widely utilized to realize the quantizer in delta-sigma modulators. However, in high-resolution implementations, the complexity of this ADC is exponentially increased. Every extra bit in such a quantizer doubles its complexity and power consumption, as well as the capacitive load of the analog circuit that drives the quantizer [1]. Accordingly, a two-step ADC quantizer is a good candidate to increase the quantizer's resolution without requiring many comparators (less loading effect) and a complex digital encoder. Such an ADC has previously been utilized in discrete-time modulators at lower sampling frequencies [7, 8]. In this work, a high-speed two-step ADC is employed to realize the quantizer of the continuous-time modulator. Figure 3 shows the two-step ADC which consists of two stages each having a 3-bit resolution. One redundant bit is present to relax the offset requirement of the comparators in the first stage. Thus, the total resolution of the quantizer is of 5 bits. The first stage is a standard switched-capacitor pipeline stage with six

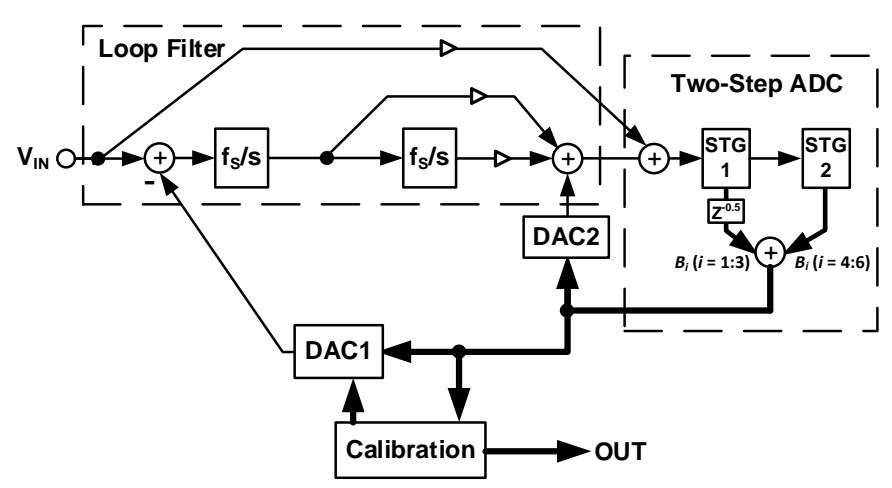

Fig. 1. The architecture of the presented 350-MS/s continuous-time delta-sigma modulator with digitally-assisted binary-DAC calibration and a 5-bits two-stepADC quantizer.

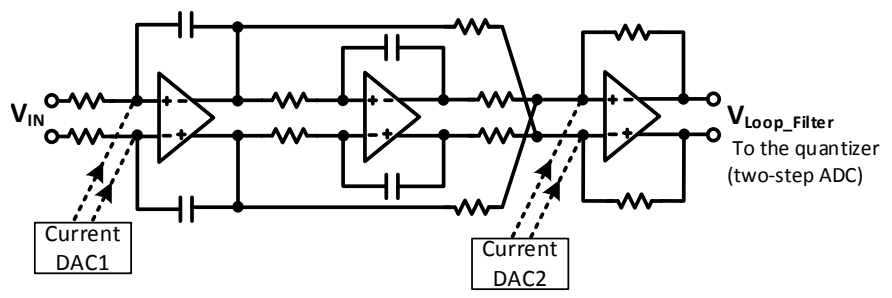

Fig. 2. The circuit details of the utilized conventional $2^{\text {nd }}$ order continuous-time loop filter consisting of two integrators and a resistive adder.

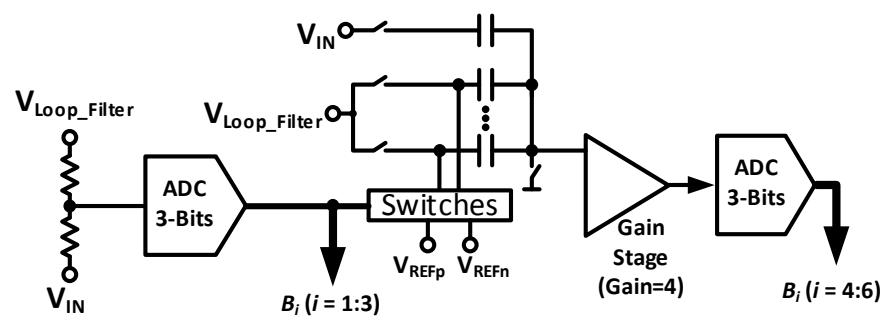

Fig. 3. The 5-bit two-step-ADC quantizer with 1-bit redundancy. The addition of the modulator's input to the loop filter output is also shown. Note that a single-ended architecture is shown for simplicity.

comparators and a capacitor-based residue-stage. The addition of the modulator's input signal at the quantizer's input seen in Fig. 1 is performed on the input capacitors of this residue stage, as shown in Fig. 3. In the residue stage, an open-loop gain stage is utilized to enhance the speed [14]. To reduce power consumption, no explicit sample and hold circuit is used. Note that the 1-bit redundancy also allows for the proposed lowcomplexity calibration technique, as described in Section 4. Note that as shown in Fig. 1, in order to synchronize the output bits of the two-step ADC, the output bits of its first stage (i.e., $\left.B_{1}-B_{3}\right)$ are delayed by a half clock cycle until the output bits of its second stage (i.e., $B_{4}-B_{6}$ ) are ready.

One of the merits of utilizing a two-step architecture is that the total quantizer delay is only slightly more than half a clock cycle. Accordingly, the instability effects resulting from this relatively short excess loop delay can be readily compensated using direct feedback through DAC2 in front of the quantizer [6, 7]. Usually, an excess loop delay that is larger than one clock-cycle is avoided since its compensation requires more complex techniques [15].

\section{Digital Calibration OF BinARY-DAC ERrors}

Traditionally, DAC nonlinearity error correction techniques 
in delta-sigma modulators are mostly based on error shaping (i.e., DEM techniques) [16]. Although these techniques are very reliable, they do not shape the DAC errors efficiently at low oversampling ratios (OSRs) [2], and they result in an extra delay in the modulator's feedback path, degrading modulator stability. Another technique to mitigate the DAC nonlinearity is calibration. Recently, different DAC calibrations in CTDS sigma modulators have been proposed particularly in low OSR modulators [2], [8]-[11]. These techniques are reported for unary DAC structures, and as such their complexity (e.g. the number of switches and their control signals, the number of unit element in the layout, etc.) can noticeably increase by the DAC resolution.

Alternatively, the digital calibration technique proposed here applies to binary current DACs and requires almost no extra analog circuitry. Note that the proposed calibration is a "self"calibration technique, since it utilizes the modulator itself to estimate the coefficient errors, which can lead to reduced area and power consumption. The digital correction block of this technique is also very simple due to the limited number of correction coefficients needed.

Figure 4a shows the utilized binary current DAC comprising of six switched current sources labeled from $I_{1}$ to $I_{6}$. Figure $4 \mathrm{~b}$ shows the transition behavior of all switch phases, $\varphi_{i}$. Here, all switch phases and their inverts are overlapped by utilizing reduced-swing high-crossing current switch drivers to minimize clock feedthrough effect and transient glitch energy $[3,17]$. During the normal operation phase of the modulator, switch phases $\varphi_{1}-\varphi_{3}$ of binary-weighted current sources $I_{1}-I_{3}$ are controlled by the output bits of the first stage of the twostep-ADC quantizer (i.e., $B_{1}-B_{3}$ ), while switch phases $\varphi_{4^{-}} \varphi_{6}$ of binary-weighted current sources $I_{4}-I_{6}$ are controlled by the output bits of the second stage of the quantizer (i.e., $B_{4}-B_{6}$ ). Here, the current of source $I_{3}$ is equal to that of source $I_{4}$ because of the 1-bit redundancy in the two-step-ADC quantizer.

The proposed foreground calibration technique estimates the relative values of current sources $I_{2}-I_{6}$ with respect to that of $I_{1}$ such that, in the digital domain, the relative bits of each current source is corrected using the estimated values.

In the calibration phase of the DAC, the input to the modulator is set to zero. Then, the values of switch phases $\varphi_{2^{-}}$ $\varphi_{6}$ of the DAC current sources are set to constants binary values. Only $\varphi_{1}$ is connected to the output of the quantizer (i.e., $B_{1}$ ). This is analogous to having a single output modulator (i.e., $B_{1}$ ), where $\varphi_{2-} \varphi_{6}$ control a current offset value, Ios, at the output of the DAC. This current can also be converted to a voltage offset at the input of the modulator. Since the input of the modulator is zero, the average of the modulator's output (i.e., $B_{1}$ ) is relative to the offset value set by $\varphi_{2}-\varphi_{6}$.

A typical calibration sequence for source $I_{2}$ is described in order to illustrate the method. First, $B_{2}$ is set to "- 1 " and the mean value of $B_{1}(M B 0)$ is saved ${ }^{1}$. Then, $B_{2}$ is set to " +1 ", and the mean value of $B_{1}(M B 1)$ is saved again. The difference between these two values (i.e., $M B 0-M B 1$ ) is equal to $I_{2} / I_{1}$. This is explained by the fact that when $B_{2}$ goes from " -1 " to " +1 ", the offset current $I_{O S}$ increases by $I_{2}$, and this offset is canceled
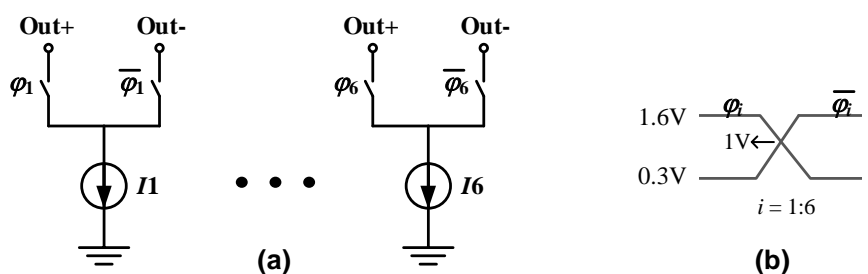

(b)

Fig. 4. (a) Utilized binary current DAC with $I_{1}=I_{R E F}, I_{2}=I_{R E F} / 2, I_{3}=I_{4}=I_{R E F} / 4$, $I_{5}=I_{R E F} / 8$, and $I_{6}=I_{R E F} / 16$. During the normal operation of the modulator, phases $\varphi_{1}-\varphi_{6}$ are connected to $B_{1}-B_{6}$, respectively. During the DAC calibration, these phases are connected as detailed in Table I. (b) The transition behavior of all switches phases, $\varphi_{i}$.

Table I. States of all phases $\varphi_{i}$ during the DAC calibration.

\begin{tabular}{c|cccccc}
\hline $\begin{array}{c}\text { Current source } \\
\text { under } \\
\text { calibration }\end{array}$ & $\varphi_{1}$ & $\varphi_{2}$ & $\varphi_{3}$ & $\varphi_{4}$ & $\varphi_{5}$ & $\varphi_{6}$ \\
\hline$I_{2}$ & $B_{1}$ & - & $-1 \rightarrow+1$ & -1 & +1 & +1 \\
$I_{3}$ & $B_{1}$ & -1 & $\begin{array}{c}- \\
1 \rightarrow 1\end{array}$ & +1 & -1 & +1 \\
$I_{4}$ & $B_{1}$ & -1 & +1 & $\begin{array}{c}- \\
1 \rightarrow+1\end{array}$ & -1 & +1
\end{tabular}

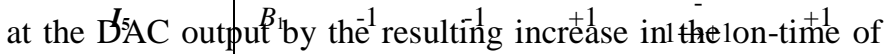
current fource $I_{-}\left(\dot{B}_{1}\right.$.e, the ${ }_{1}$ code density $a_{1} B_{1}=1$ is increased $_{1}$ in relationto $\left.T_{2} / I_{1}\right)$. For added detall, the mathematical calculations of this calibration method are presented in Appendix A. Using the same procedure, the relative values of the other current sources with respect to that of $I_{1}$ can be estimated. Note that the offset value of the ADC does not affect this estimation since its value affects both $M B 0$ and $M B 1$, and hence it is canceled when calculating $M B 0-M B 1$.

During the calibration phase, the modulator effectively utilizes a 1-bit quantizer, which limits its output linear range. If the current source switches are not set properly such that $M B 0$ and $M B 1$ are symmetric (i.e., $M B 1 \approx-M B 0$ ), the value of either $M B 0$ or $M B 1$ can more likely become sufficiently large to come close to the output linear range of the modulator such that the value of $M B 0-M B 1$ will include some error. Accordingly, when a current source is under calibration, the other current source switches are set such that the values of $M B 0$ and $M B 1$ are symmetric to help estimate the values of $M B 1$ and $M B 0$ more accurately. This can be done by setting the current source switches as shown in Table I. Interestingly, this symmetry is made possible by the use of redundant bits $B_{3}$ and $B_{4}$ in the quantizer, and it could not be realized with a standard binary output.

As can be seen, this calibration technique does not add any extra analog circuitry to the ADC as only a few switches are added to the DAC, and do not affect its behavior during its normal operation. Note that a calibration technique based on similar toggling of the DAC unit elements is also presented in [18] for a discrete-time delta-sigma modulator. However, it utilizes extra DAC elements to estimate the coefficient errors, and not the modulator DAC itself. As demonstrated in Section $\mathrm{V}$, the proposed calibration technique can estimate a relatively large current-mismatch value of $5 \%$. 


\section{MEASUREMENT RESUlTS}

The $2^{\text {nd }}$-order continuous-time delta-sigma modulator that utilizes the proposed techniques was implemented in a $130-\mathrm{nm}$ CMOS technology. A chip micrograph is shown in Fig. 5. The circuitry occupies an active area of $260 \mu \mathrm{m} \times 300 \mu \mathrm{m}$. Measurements of this proof-of-concept prototype demonstrated that the proposed calibration technique can calibrate up to 5\% of mismatch in the DAC elements. Note that, in this design, the DAC elements were sized intentionally small to be relatively highly mismatched. Table II shows the DAC mismatch values that were estimated using the proposed calibration technique.

As shown in Fig. 6a before calibration, the measurements of the modulator show an SNDR, SNR and SFDR of $35.5 \mathrm{~dB}$, $40.4 \mathrm{~dB}$ and $40.9 \mathrm{~dB}$, respectively at an $8.75 \mathrm{MHz}$ bandwidth (sampling rate of $350 \mathrm{MS} / \mathrm{s}$ and OSR of 20). As shown in Fig. 6b, with the same bandwidth, the proposed calibration technique improves the SNDR, SNR and SFDR values to $60.3 \mathrm{~dB}, 62 \mathrm{~dB}$ and $74 \mathrm{~dB}$, respectively. Moreover, the modulator's dynamic range is $65 \mathrm{~dB}$ after calibration, as can be seen in Fig. 7. The total power consumption of the circuit is $5.5 \mathrm{~mW}$ from a $1.6 \mathrm{~V}$ supply voltage.

Table III lists the performance summary of the presented CTDS modulator, outlining its main conversion characteristics and its performance before and after calibration. Figure 8 shows a comparison of the presented CTDS modulator to state-of-theart CTDS modulators in $130 \mathrm{~nm}$ CMOS. The following figure of merit (FOM) is used here:

$$
F O M=\frac{\text { Power }}{2 \times B W \times 2^{E N O B}},
$$

where $B W$ represents the modulator bandwidth, and ENOB the effective number of bits. Although the goal of this work is to provide a proof-of-concept design for the two presented techniques, its figure-of merit (FOM) remains comparable to the state-of-the-art CTDS modulators in $130 \mathrm{~nm}$ CMOS, while providing advantages with regards to design simplicity in the increased-resolution quantizer and increased-resolution DAC, and with regards to robustness against high mismatch values in the DAC elements.

It should be noted that the modulator exhibits higher bandwidth and resolution in simulations. However, due to some unforeseen issues in its digital block, the sampling frequency had to be reduced. This reduction resulted in a lower modulator bandwidth, and also affected the noise shaping behavior of the modulator, which reduced the effective measured resolution. Furthermore, a few missing codes in the output of the two-step ADC were observed during measurements. This slight reduction in the quantizer's resolution, attributed to process variations, can lead to a reduced performance of the modulator. However, this is mitigated by the shaping of the quantizer's error by the loop filter, and its effect is accounted for in the measurement results.

\section{CONCLUSION}

This paper presented a proof-of-concept prototype in 130-nm CMOS to introduce two techniques to improve continuous-time delta-sigma modulators. A low-complexity calibration technique is introduced to digitally estimate and correct the errors in a

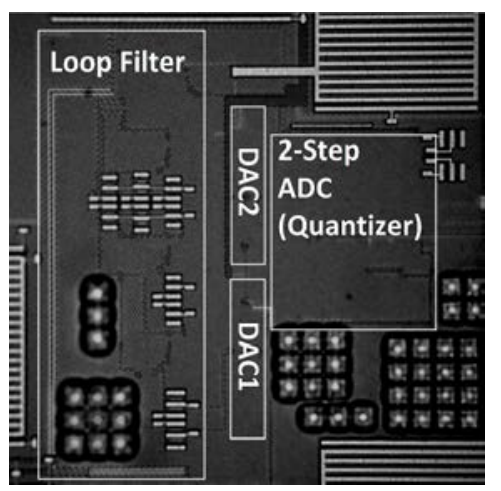

Fig. 5. Chip micrograph of the fabricated die in $130 \mathrm{~nm}$ CMOS with an active area of $260 \mu \mathrm{m} \times 300 \mu \mathrm{m}$.

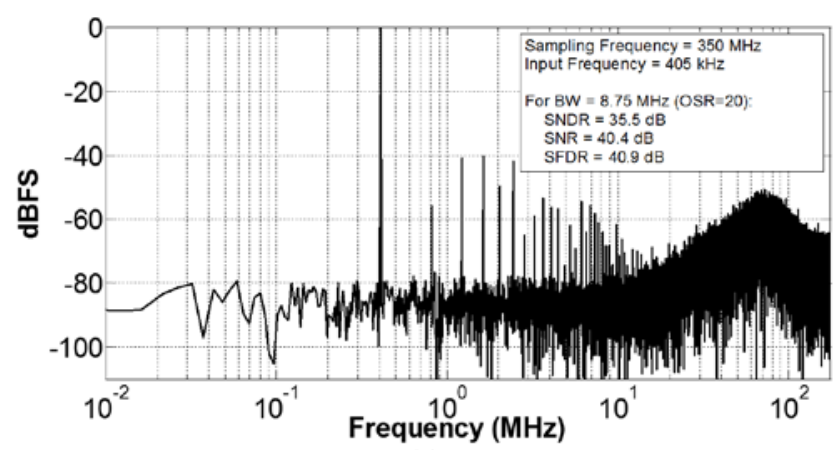

(a)

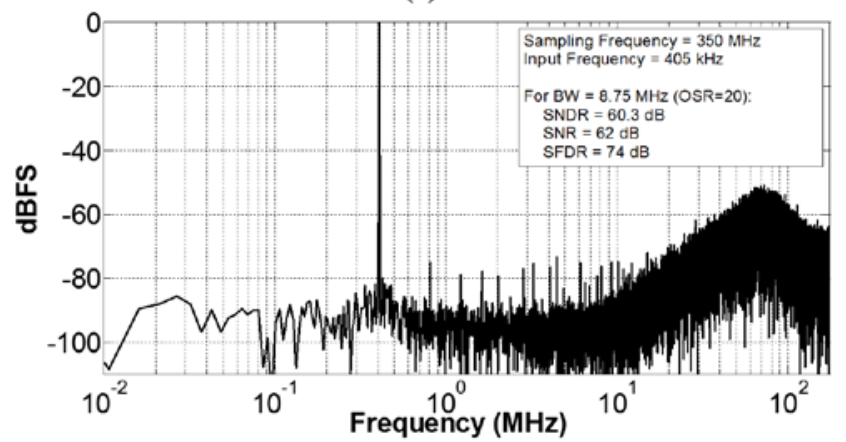

(b)

Fig. 6. The output spectrum of the presented CTDS modulator (a) before calibration, and (b) after calibration.

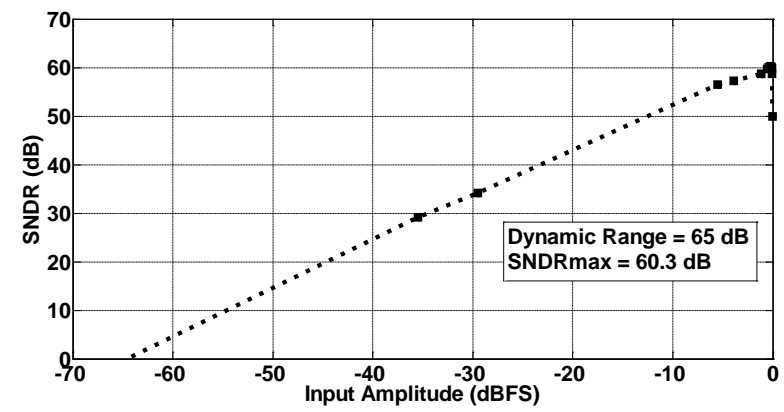

Fig. 7. Measured output SNDR versus the input amplitude of the modulator.

Table II. Estimated values of the DAC elements during measurements of a typical die using the proposed calibration technique.

\begin{tabular}{l|ccccc}
\hline \multicolumn{1}{c}{} & $\boldsymbol{I}_{\mathbf{2}} / \boldsymbol{I}_{\mathbf{1}}$ & $\boldsymbol{I}_{\mathbf{3}} / \boldsymbol{I}_{\mathbf{1}}$ & $\boldsymbol{I}_{\mathbf{4}} / \boldsymbol{I}_{\mathbf{1}}$ & $\boldsymbol{I}_{\mathbf{5}} / \boldsymbol{I}_{\mathbf{1}}$ & $\boldsymbol{I}_{\mathbf{6}} / \boldsymbol{I}_{\mathbf{1}}$ \\
\hline Ideal Value & 0.5 & 0.25 & 0.25 & 0.125 & 0.0625 \\
Estimated Value & 0.4731 & 0.2533 & 0.2368 & 0.1233 & 0.06318 \\
Error (\%) & -5.3 & +1.3 & -5.2 & -1.3 & +1.1 \\
\hline
\end{tabular}


Table III. Performance summary of the presented CTDS modulator.

\begin{tabular}{|c|c|c|}
\hline \multicolumn{2}{|l|}{ Parameter } & Value \\
\hline \multicolumn{2}{|l|}{ Technology } & 130 nm CMOS \\
\hline \multicolumn{2}{|l|}{ Supply Voltage } & $1.6 \mathrm{~V}$ \\
\hline \multicolumn{2}{|c|}{ Sampling Frequency } & $350 \mathrm{MS} / \mathrm{s}$ \\
\hline \multicolumn{2}{|c|}{ Input Range (differential) } & $1.8 \mathrm{~V}$ \\
\hline $\begin{array}{l}\text { SNDR / SFDR } \\
(\mathrm{OSR}=20)\end{array}$ & $\begin{array}{l}\text { Before cal. } \\
\text { After cal. }\end{array}$ & $\begin{array}{c}35.5 \mathrm{~dB} / 50.9 \mathrm{~dB} \\
60.3 \mathrm{~dB} / 74 \mathrm{~dB}\end{array}$ \\
\hline \multicolumn{2}{|c|}{$\begin{array}{l}\text { SNR / Dynamic Range } \\
\text { after calibration (OSR=20) }\end{array}$} & $62 \mathrm{~dB} / 65 \mathrm{~dB}$ \\
\hline \multicolumn{2}{|c|}{ Total Power Consumption } & $5.5 \mathrm{~mW}$ \\
\hline \multicolumn{2}{|l|}{ Total Area } & $260 \mu \mathrm{m} \times 300 \mu \mathrm{m}$ \\
\hline \multicolumn{2}{|l|}{ FOM } & 0.37 pJ/Conv-step \\
\hline
\end{tabular}

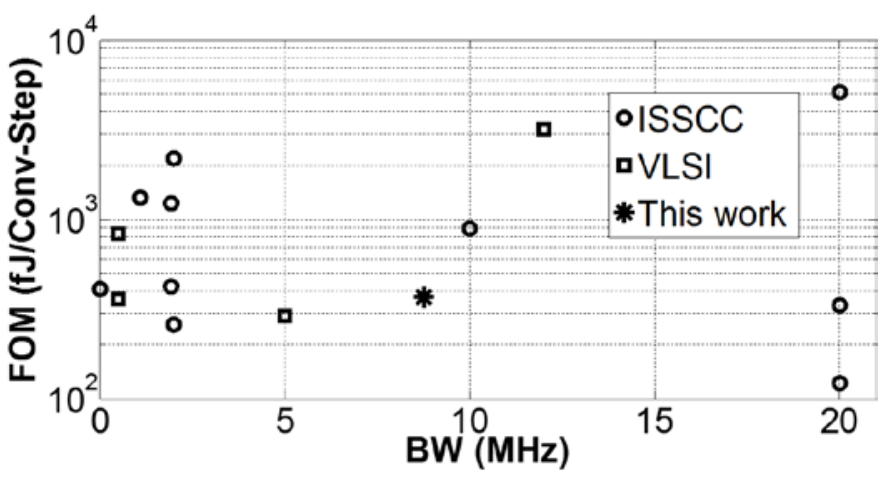

Fig. 8. Comparison of this work to state-of-the-art delta-sigma modulators implemented in $130 \mathrm{~nm}$ CMOS that are reported in the International Solid-State Circuits Conference (ISSCC) or the Symposium on VLSI Circuits (VLSI) [19].

binary current DAC. Thus, no relatively complex dynamic element matching (DEM) technique is required, and the related delay and power consumption increase is avoided. The proposed calibration technique can calibrate up to a $5 \%$ of mismatch in the DAC elements with very little added circuitry and additional active area. Furthermore, for continuous-time delta-sigma modulators with high-sampling rates, a high-speed two-stepADC quantizer is introduced to efficiently increase the modulator's resolution. The modulator has a measured SNDR of $60.3 \mathrm{~dB}$ and an SFDR of $74 \mathrm{~dB}$ for a sampling rate of $350 \mathrm{MS} / \mathrm{s}$ and oversampling ratio (OSR) of 20 , translating to a $8.75 \mathrm{MHz}$ bandwidth.

Ultimately, the CTDS modulator introduced in this work enables the use of a low-complexity quantizer with increased resolution and of a DAC with increased resolution that is calibrated for high mismatch values in its elements.

\section{ACKNOWLEDGEMENT}

The authors would like to thank the late Prof. Anas Hamoui (McGill University) for the fruitful discussions.

\section{REFERENCES}

[1] J. De Maeyer, P. Rombouts, and L. Weyten, "Efficient multibit quantization in continuous-time Sigma Delta modulators,” IEEE Trans. Circuits Syst.-I, vol. 54, no. 4, pp. 757 767, Apr. 2007.

[2] J. G. Kauffman, P. Witte, J. Becker, and M. Ortmanns, "An 8.5 mW Continuous-Time Delta-Sigma Modulator With $25 \mathrm{MHz}$ Bandwidth
Using Digital Background DAC Linearization to Achieve $63.5 \mathrm{~dB}$ SNDR and 81 dB SFDR,” ," IEEE J. Solid-State Circuits, vol. 46, no. 12, pp. 2869-2881, Dec.2011.

[3] S. Yan and E. Sánchez-Sinencio, "A Continuous-Time $\Sigma \Delta$ Modulator With 88-dB Dynamic Range and 1.1-MHz Signal Bandwidth,” IEEE J. Solid-State Circuits, vol. 39, no. 1, pp. 75-86, Jan. 2004.

[4] A. A. Hamoui and K. W. Martin, "High-order multibit modulators and pseudo data-weighted-averaging in low over sampling ADCs for broadband applications," IEEE Trans. Circuits Syst. I, vol. 51, no. 1, pp. 72 85, Jan. 2004.

[5] Seung-Chul Lee and Yun Chiu, "Digital Calibration of Capacitor Mismatch in Sigma-Delta Modulators," IEEE Transactions on Circuits and Systems I, vol. 58, no. 4, pp. 690 - 698, Apr. 2011.

[6] M. Ortmanns and F. Gerfers, Continuous-time sigma-delta $A / D$ conversion: Fundamentals, performance limits and robust implementations. Netherlands: Springer Berlin Heidelberg, 2006.

[7] F. Ali and A. A. Hamoui, "Continuous-time $\Delta \Sigma$ modulators with noisetransfer-function enhancement," in IEEE International Symposium on Circuits and Systems (ISCAS), May 2008, pp. 1428-1431.

[8] C.-L. Lo, C.-Y. Ho, H.-C. Tsai, and Y.-H. Lin, "A 75.1dB SNDR $840 \mathrm{MS} / \mathrm{s}$ CT $\Delta \Sigma$ Modulator with $30 \mathrm{MHz}$ Bandwidth and $46.4 \mathrm{fJ} / \mathrm{conv}$ FOM in 55nm CMOS," IEEE Symposium on VLSI circuits, 2013, pp. C60-C61.

[9] X. Xing, M. De Bock, P. Rombouts, and G. Gielen, “A 40MHz 12bit 84.2dB-SFDR Continuous-Time Delta-Sigma Modulator in $90 \mathrm{~nm}$ CMOS,” IEEE Asian Solid-State Circuits Conference, 2011, pp. 249252.

[10] M. De Bock, X. Xing, L.Weyten, G. Gielen, and P. Rombouts, "Calibration of DAC Mismatch Errors in $\Sigma \Delta$ ADCs Based on a SineWave Measurement," IEEE Transactions on Circuits and Systems II, vol. 60, no. 9, pp. 567 - 571, Sept. 2013.

[11] Z. Li and T. S. Fiez, "A 14-Bit Continuous-Time Delta Sigma A/D Modulator with $2.5 \mathrm{MHz}$ Signal Bandwidth,” IEEE J. Solid-State Circuits, vol. 42, no. 9, pp. 1873-1883, Sept. 2007.

[12] O. Rajaee, S. Takeuchi, M. Aniya, K. Hamashita, and U. Moon, "LowOSR over-ranging hybrid ADC incorporating noise-shaped two-step quantizer," IEEE J. Solid-State Circuits, vol. 46, no. 11, pp. 2458-2468, Nov. 2011.

[13] O. Rajaee, T. Musah, N. Maghari, S. Takeuchi, M. Aniya, K. Hamashita, and U. Moon, "Design of a $79 \mathrm{~dB} 80 \mathrm{MHz} 8 \mathrm{X}-$ OSR hybrid deltasigma/pipeline ADC," IEEE J. Solid-State Circuits, vol. 45, no. 4, pp. 719-730, Apr. 2010.

[14] B. Murmann and B. Boser, "A 12-bit 75-MS/s pipelined ADC using open-loop residue amplification,” IEEE J. Solid-State Circuits, vol. 38, no. 12, pp. 2040-2050, Dec. 2003.

[15] V. Singh, N. Krishnapura, S. Pavan, B. Vigraham, D. Behera, N. Nigania, "A $16 \mathrm{MHz}$ BW $75 \mathrm{~dB}$ DR CT $\Delta \Sigma$ ADC Compensated for More Than One Cycle Excess Loop Delay," IEEE J. Solid-State Circuits, vol. 47, no. 8, pp. 1884-1895, Aug. 2012

[16] L. R. Carley, "A noise-shaping code topology for 15+ bit converters," IEEE J. Solid-State Circuits, vol. 24, no. 4, pp. 267-273, Apr. 1989.

[17] K. Falakshahi, C.-K. K. Yang, and B. A. Wooley, "A 14-bit, 10Msamples/s D/A converter using multibit sigma-delta modulation," IEEE J. Solid-State Circuits , vol. 34, pp. 607-615, May 1999.

[18] M. Sarhangnejad and G. Temes, "A high-resolution multibit-SigmaDelta ADC with digital correction and relaxed amplifier requirements," IEEE J. Solid-State Circuits, vol. 28, no. 6, pp. 648 660, Jun. 1993.

[19] B. Murmann, “ADC Performance Survey 1997-2013," [Online]. Available: http://www.stanford.edu/ murmann/adcsurvey.html.

\section{APPENDIX A}

In summary, in the proposed DAC calibration technique, by two consecutive measurements in which the code of $B_{2}$ is toggled, the ratio of $I_{2} / I_{1}$ is extracted. This appendix aims at briefly presenting the mathematical details of the proposed calibration technique.

Toggling code $B_{2}$ from "- 1 " to “+1", is equivalent to adding an error signal to the DAC output with a normalized value of

$$
E_{D A C}=\frac{2 I_{2}}{I_{R E F}}
$$


where $I_{R E F}$ is the differential reference current of the DAC. Since the DAC is a single-bit DAC with $I_{R E F}=2 I_{1}$,

$$
E_{D A C}=\frac{I_{2}}{I_{1}} .
$$

This error signal goes to the modulator's output through the DAC error transfer function of:

$$
\operatorname{ETF}(z)=\frac{H(z)}{1+H(z)}
$$

where $H(z)$ is the equivalent loop-filter transfer function (in discrete-time domain) of the modulator. Since during the calibration phase, $V_{I N}=0$, the modulator's output is given by

$$
\begin{aligned}
\operatorname{OUT}(z) & =\operatorname{NTF}(z) \times Q_{N}(z)+\operatorname{ETF}(z) \times E_{D A C} \\
& =\frac{1}{1+H(z)} Q_{N}(z)+\frac{H(z)}{1+H(z)} \frac{I_{2}}{I_{1}}
\end{aligned}
$$

where $Q_{N}$ is the quantization noise of the quantizer. Considering a high low-frequency gain for $H$, the DC value of the modulator's output is equal to $I_{2} / I_{1}$. Similar technique can be used to measure other current source values.

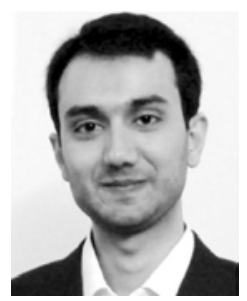

Mohammad Taherzadeh-Sani received his B.Sc. from Ferdowsi University of Mashhad, Iran, in 2001, his M.Sc. from the University of Tehran, Iran, in 2004, and his Ph.D. degree from McGill University, Montreal, Canada, in 2011. He was awarded J.W. McConnell Memorial Fellowship from McGill University in 2007 and 2008 for his doctoral research. He was also awarded a competitive postdoctoral fellowship from the Le Fonds Québécois de la Recherche sur la Nature et les Technologies (FQRNT) for 2012 and 2013 (declined).

In 2012, he joined Ferdowsi University of Mashhad, Mashhad, Iran, as an Assistant Professor. His research interests focuses on biomedical circuits and systems, high-quality and high-speed data converters, and radio frequency integrated circuits. Dr. Taherzadeh-Sani has different fabricated ICs and publications on these subjects. He fabricated several integrated circuits in various technologies from $65 \mathrm{~nm}$ to $180 \mathrm{~nm}$ CMOS. He authored several journal publications in distinguished journals (e.g., JSSC, TCAS-I, TCAS-II, and T-VLSI) and many conference papers in well-known conferences (e.g., ESSCIRC, A-SSCC, ICCAD, and ISCAS).

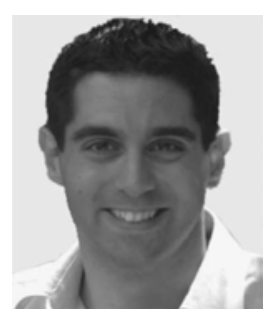

Frederic Nabki (S'99-M'10) received the B.Eng. degree in electrical engineering with Honors from McGill University, Montreal, QC, Canada, in 2003, and the Ph.D. degree in electrical engineering from McGill University in 2010.

In 2008, he joined the "Université du Québec à Montréal” (UQAM), Montreal, QC, Canada, where he is currently an Associate Professor in microelectronics engineering. His research interests include mixed-signal and radiofrequency integrated circuits and microelectromechanical systems (MEMS) for various applications such as sensing and communications. Some research projects include the design of novel low-power and high speed analog-to-digital converters, CMOS phase-locked loops, ultra-wideband transceivers, and wired communication channels. In addition, Dr. Nabki is active in research projects involving the creation of next generation MEMS fabrication processes using advanced materials, the integration of MEMS devices with CMOS systems, and the modeling of MEMS devices.

Dr. Nabki is a member of the Quebec Order of Engineers. He was a recipient of the Governor General of Canada's Academic Bronze Medal. He has held support from the Microsystems Strategic Alliance of Quebec (ReSMiQ), the Quebec Fund for Research in Nature and Technology (FRQ-NT), the Natural Sciences and Engineering Research Council of Canada (NSERC), and the Canada Foundation for Innovation (CFI). 